\title{
Treating osteonecrosis with autologous bone marrow cells
}

\author{
Valérie Gangji • Jean-Philippe Hauzeur
}

Published online: 17 September 2009

(C) ISS 2009

Osteonecrosis is a devastating disease that primarily affects weight-bearing joints. The hip is the most commonly affected joint. Although osteonecrosis of the femoral head can affect patients of any age group, it typically presents in young patients between the ages of 20 and 40 . The evolution of osteonecrosis is towards femoral head collapse, osteoarthritis and finally total hip replacement. Osteonecrosis is believed to be a multifactorial disease that is associated in some cases with both genetic predilection and exposure to certain risk factors. These risks factors include corticosteroid use, alcohol intake, smoking and various chronic diseases such as renal disease, haematological disease, inflammatory bowel disease, post-organ transplantation, systemic lupus erythematosus and human immunodeficiency virus. However, in Caucasians, corticosteroids and alcohol abuse are among the most widely recognized risk factors for osteonecrosis. In some cases, osteonecrosis of the femoral head has also been associated with certain genetic polymorphisms such as alcoholmetabolizing enzymes and the drug-transport protein Pglycoprotein. Liu et al. also identified three families with an autosomal dominant inheritance of osteonecrosis of the femoral head and mapped the chromosomal position of the collagen type-II gene (COL2A1 gene) mutation [1]. The importance of those findings is that genetic screening of

\section{Gangji $(\bowtie) \cdot$ J.-P. Hauzeur}

Rheumatology and Physical Medicine Department,

Erasme Hospital, Université Libre de Bruxelles,

808 Route de Lennik,

1070 Bruxelles, Belgium

e-mail: valerie.gangji@erasme.ulb.ac.be

J.-P. Hauzeur

Rheumatology Department, CHU Sart Tilman,

4000 Liège, Belgium families with osteonecrosis could be used to identify carriers before the onset of clinical symptoms and screening with magnetic resonance imaging (MRI) could enable an early diagnosis and the initiation of measures that could delay disease progression. Magnetic resonance imaging is important in the diagnosis of hip osteonecrosis as well as in predicting the outcome. Standard radiographs are commonly used to stage the progression of the disease. The factors that affect the progression of this disease are still not fully understood but lesion size, stage of disease (femoral head collapse), time from the diagnosis and etiological factors have been shown to be predictive of the clinical outcome. The most important factor in predicting the outcome of osteonecrosis is the size of the necrotic lesion [2]. The lesion size can be estimated radiographically when evident and by MRI during early stages of the disease. Different methods have been assessed radiographically and by MRI to measure the amount of femoral head involvement by osteonecrosis (calculation of necrotic angle, of necrotic index, estimation of percent of involvement and exact volumetric analysis). They can all be used with confidence as they are highly reliable and reproductible.

There continues to be a lack of consensus concerning the pathogenesis and treatment of the disease despite recent outstanding studies and reviews. Different mechanisms leading to ischemia have been postulated including fat emboli, microvascular tamponade of the blood vessels of the femoral head by marrow fat, retrograde embolization of the marrow fat and intravascular coagulation. However, none of those mechanisms explored the necrotic lesion as a primary bone disease. Indeed, osteonecrosis is characterized by apoptosis of osteocytes and cancellous bone lining cells in the necrotic lesion and also at some distance from the lesion, in the proximal femur [3]. The replicative capacities of osteoblastic cells obtained from the intertrochanteric area 
of the femur are reduced in patients with osteonecrosis [4]. The number and the activity of fibroblast colony-forming units, reflecting the number of mesenchymal stem cells that could potentially give rise to mature osteoblasts have been shown to be decreased in osteonecrosis $[5,6]$. Moreover, the capillaries serving as a conduit for the stem cells and bone cells needed in bone repair for osteonecrosis in addition to providing blood supply are altered by emboli or thrombosis [7]. Osteonecrosis could therefore be seen as both a vascular and bone disease. The altered bone remodeling process appears to be responsible for three different events in the pathogenesis of osteonecrosis; the appearance of the necrotic segment, the altered bone repair that occurs after osteonecrosis and its evolution to subchondral fracture. Glucocorticoids inhibit osteoblastogenesis and promote osteoblast and osteocyte apoptosis $[8,9]$. Apoptosis could disrupt the mechanosensory role of osteocytes and thus prevent the adaptation of bone to ischemia [9-11]. The decrease in osteoblast capacity to proliferate could therefore reflect the disruption of the mechanosensory role of the osteocytes canalicular network and explain the progression from marrow ischemia and oedema to osteonecrosis [4]. Therefore, a sufficient repair capability at an early stage would make the lesion reversible. An insufficient repair mechanism, as seen in osteonecrosis, related to a decrease in bone formation might explain the advancement to a further stage of osteonecrosis and eventual subchondral fracture. The rate of bone formation is indeed largely determined by the number of osteoblasts, which, in turn, is determined by the rate of replication of progenitors [12]. The improved capillary function will enable stem cells to travel from the bone marrow to the bone surface to meet the need for bone remodeling necessary to heal the necrotic lesion.

In the natural evolution of osteonecrosis, most early stages osteonecrotic lesion (stage-I or -II) will evolve to fracture (stage-III) and collapse of the femoral head. Symptomatic osteonecrosis of the femoral head generally progresses within 24 months to subchondral fracture, collapse and eventually painful osteoarthritis of the hip. At present, most patients with symptomatic osteonecrosis with collapse undergo total hip replacement. Indeed, over the past 10 years, multiple studies have demonstrated excellent rates of success of total hip arthroplasty in patients with osteonecrosis. However, because of the young age of some of these patients, a hip replacement cannot be expected to last the patient's lifetime and therefore, when feasible, attempts should be made to protect the femoral head from collapse with the use of less invasive modalities. The efficacy of these procedures has been variable, with reported success rates ranging from $60 \%$ and $80 \%$ at shortterm and midterm follow-up. Current treatments range from pharmacotherapies to surgical interventions that include core decompression, vascularized or nonvascularized bonegrafting and osteotomy. Core decompression is still the most widespread procedure to treat early stages of osteonecrosis of the femoral head. Notwithstanding the fact that this procedure has been used for more than four decades, its efficacy remains controversial. Indeed, in randomized controlled trials, the efficacy of core decompression measured in terms of decreased proportion of patients having additional surgeries or showing delayed radiological progression to collapse could not be demonstrated. Therefore, there have been attempts to treat osteonecrosis by implantation of stem cells into the necrotic lesion or by utilizing growth factors [13]. The rationale for this approach is based on our present pathophysiological understanding of the sequence of events in osteonecrosis.

Autologous bone-marrow transplantation was reported for the first time in a patient suffering from osteonecrosis of the humeral head due to sickle cell anemia with improvement of pain and range of motion and reconstruction of the humeral epiphysis [14]. Thereafter, Hernigou et al. and Gangji et al. studied the efficacy of bone marrow implantation into the necrotic lesion of osteonecrosis of the femoral head [16]. Four hundred millilitres of bone marrow is aspirated from the iliac crest with the patient under general anesthesia. Mononuclear cells are then isolated on a cell separator and concentrated to a final volume of $50 \mathrm{ml}$. Under fluoroscopic control, a $3 \mathrm{~mm}$ trephine is inserted manually through the trochanter, the neck and the head of the femur to the necrotic lesion. The tip of the trephine is placed in the necrotic lesion at a distance of 2-3 mm from the articular cartilage. The $50 \mathrm{ml}$ of mononuclear cells are then injected through the trephine that is placed into the necrotic lesion. The injection of $50 \mathrm{ml}$ is possible since the necrotic zone contains intertrabecular spaces that can be filled with bone marrow. After the implantation, under fluoroscopic guidance, the trephine is removed and sutures applied. Patients remain non-weight-bearing on the operative side for 3 weeks, after which total weight bearing is permitted.

Gangji et al. studied 13 patients (eighteen hips) with stage-I or II (without subchondral fracture) osteonecrosis of the femoral head according to the Association Research Circulation Osseous classification in a controlled double blind trial with 24 months of follow-up [15]. The etiological factors were corticosteroid therapy and alcohol abuse. The hips were allocated to a program of core decompression only (control group) or core decompression and implantation of bone marrow mononuclear cells (bone marrow graft group). After a 24-month follow up, there was a significant reduction in pain and joint symptoms within the bone marrow graft group $(p=0.021)$. At 24 months, 5 of the 8 hips in the control group had deteriorated to stage-III (stage of the subchondral fracture), whereas only one of the 
10 hips in the bone marrow graft group had progressed to this stage $(p=0.016)$. Survival analysis showed a significant difference in the time to collapse between the two groups. Moreover, in the bone marrow graft group, the volume of the necrotic lesion decreased by $35 \%$ after 24 months. Similarly, Hernigou and Beaujean reported the results of a prospective study of 189 hips in 116 patients treated with core decompression and bone marrow grafting [16]. The patients were followed up from 5 to 11 years with a mean of 7 years. The etiological factors were corticosteroids, alcohol abuse, sickle cell disease and organ transplantation. The outcome measures were changes in clinical symptoms, progression in radiographic stages and the need for hip replacement. When patients were treated before collapse (stage-I and II), hip replacement was done in 9 of the 145 hips. Total hip replacement was necessary in 25 hips among the 44 hips operated after collapse (stage-III and IV). The number of mesenchymal stems cells implanted might have had an influence on the outcome of the hip. Altogether, the two studies showed improvement in femoral head preservation after implantation of stem cells in early osteonecrosis of the femoral head.

There are a number of patient specific factors that might be considered when determining the outcome of the disease and the treatment such as risk factors, lesion size, age, life expectancy health comorbidities and activity level. However, new insights into the pathophysiology of osteonecrosis as a concurrent bone and vascular disease and treatment modifications based on this understanding show promise in the arrest of complications from early osteonecrosis.

\section{References}

1. Liu YF, Chen WM, Lin YF, Yang RC, Lin MW, Li LH, et al. Type II collagen gene variants and inherited osteonecrosis of the femoral head. N Engl J Med. 2005;352(22):2294-301.

2. Nam KW, Kim YL, Yoo JJ, Koo KH, Yoon KS, Kim HJ. Fate of untreated asymptomatic osteonecrosis of the femoral head. J Bone Jt Surg Am. 2008;90(3):477-84.
3. Calder JD, Pearse MF, Revell PA. The extent of osteocyte death in the proximal femur of patients with osteonecrosis of the femoral head. J Bone Jt Surg Br. 2001;83(3):419-22.

4. Gangji V, Hauzeur JP, Schoutens A, Hinsenkamp M, Appelboom T, Egrise D. Abnormalities in the replicative capacity of osteoblastic cells in the proximal femur of patients with osteonecrosis of the femoral head. J Rheumatol. 2003;30(2):348-51.

5. Hernigou P, Beaujean F. Abnormalities in the bone marrow of the iliac crest in patients who have osteonecrosis secondary to corticosteroid therapy or alcohol abuse. J Bone Jt Surg Am. 1997;79(7):1047-53.

6. Hernigou P, Beaujean F, Lambotte JC. Decrease in the mesenchymal stem-cell pool in the proximal femur in corticosteroidinduced osteonecrosis. J Bone Jt Surg Br. 1999;81(2):349-55.

7. Simkin PA, Downey DJ. Hypothesis: retrograde embolization of marrow fat may cause osteonecrosis. J Rheumatol. 1987;14(5):870-2.

8. O'Brien CA, Jia D, Plotkin LI, Bellido T, Powers CC, Stewart SA, et al. Glucocorticoids act directly on osteoblasts and osteocytes to induce their apoptosis and reduce bone formation and strength. Endocrinology. 2004;145(4):1835-41.

9. Weinstein RS, Jilka RL, Parfitt AM, Manolagas SC. Inhibition of osteoblastogenesis and promotion of apoptosis of osteoblasts and osteocytes by glucocorticoids: potential mechanisms of their deleterious effects on bone. J Clin Invest 1998 July 15;102 (2):274-82.

10. Hauzeur JP, Perlmutter N, Appelboom T, Pasteels JL. Medullary impairment at early stage of non-traumatic osteonecrosis of the femoral head. Rheumatol Int. 1991;11(4-5):215-7.

11. Weinstein RS, Nicholas RW, Manolagas SC. Apoptosis of osteocytes in glucocorticoid-induced osteonecrosis of the hip. J Clin Endocrinol Metab. 2000;85(8):2907-12.

12. Shih MS, Cook MA, Spence CA, Palnitkar S, McElroy H, Parfitt AM. Relationship between bone formation rate and osteoblast surface on different subdivisions of the endosteal envelope in aging and osteoporosis. Bone. 1993;14(3):519-21.

13. Lieberman JR, Conduah A, Urist MR. Treatment of osteonecrosis of the femoral head with core decompression and human bone morphogenetic protein. Clin Orthop Relat Res 2004 December; (429):139-45.

14. Hernigou P, Bernaudin F, Reinert P, Kuentz M, Vernant JP. Bonemarrow transplantation in sickle-cell disease: effect on osteonecrosis: a case report with a four-year follow-up. J Bone Jt Surg Am. 1997;79(11):1726-30.

15. Gangji V, Hauzeur JP, Matos C, De MV, Toungouz M, Lambermont M. Treatment of osteonecrosis of the femoral head with implantation of autologous bone-marrow cells: a pilot study. J Bone Joint Surg Am. 2004;86-A(6):1153-60.

16. Hernigou P, Beaujean F. Treatment of osteonecrosis with autologous bone marrow grafting. Clin Orthop 2002 December; (405):14-23. 